\title{
Correction to "Surface deformation due to a strike-slip fault in an elastic gravitational layer overlying a viscoelastic gravitational half-space" by Ting-To Yu, John B. Rundle, and José Fernández
}

In the paper "Surface deformation due to a strike-slip fault in an elastic gravitational layer overlying a viscoelastic gravitational half-space" by Ting-To Yu, John B. Rundle, and José Fernández (Journal of Geophysical Research, 101 (B2), 3199-3214, 1996), we have found errors while using the methods for calculating deformations produced by rectangular strike-slip fault in the described viscoelastic-gravitational layered Earth.

$\mathrm{Yu}$ et al. calculated the coseismic displacements by using the formulae described by Okada [1985]. However, the reference frame system used in the paper is different to the one used by Okada [1985], especially in the $Y$ component, as it is shown in Figure 1. The transformation between both systems is straightforward; see Figures 1 and 2 of correction by Fernández et al. [1996a]. Using the subscript $O$ to refer to Okada's reference system and the subscript $R$ for the system used in paper (which is the same as the one used by Rundle [1982], Fernández et al. [1996a, b], and Yu et al. [1996]), Fernández et al. [1996a] obtain

$$
\begin{aligned}
& X_{O}=X_{R} \\
& Y_{O}=-Y_{R}+(W+D / \sin \psi) \cos \psi \\
& Z_{O}=-Z_{R}
\end{aligned}
$$

The calculations described by $\mathrm{Yu}$ et al. mistakenly showed $Y_{R}=Y_{O}$. As a consequence, in their figures, only the coseismic displacements due to strike-slip faulting have such error. Another mistake is the singularity problem in using Okada's definition. There are two kinds of singularity conditions that happened within the code. One will cause the function reach to zero, while another one will lead the values of function becomes to infinite. However, only the first kind of situation is considered within the original case. For all that reasons, coseismic displacements shown

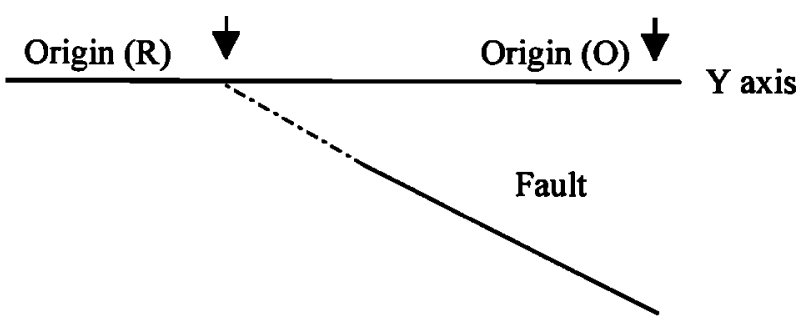

Figure 1. Definition of the origins for $Y$ coordinate at Okada [1985] (O) and Rundle [1982] (R) systems.

Copyright 1999 by the American Geophysical Union.

Paper number $1999 \mathrm{JB} 900119$.

0148-0227/99/1999JB900119\$09.00 in figures by $\mathrm{Yu}$ et al. are not correct. The corrected plots are shown as Figures 2 to 8 . In this case, the error does not affect the concluded result of papers, which are solely based upon the viscoelastic displacements, and this result is calculated with the reference system used by Yu et al. directly; thus the reason it is not affected by using this incorrect transformation of reference frame.

Also Yu et al.'s equation (15) contain some minor misprints and should be replaced by the following:

Horizontal strike-slip fault $\left(X=200, \theta=90^{\circ}\right)$
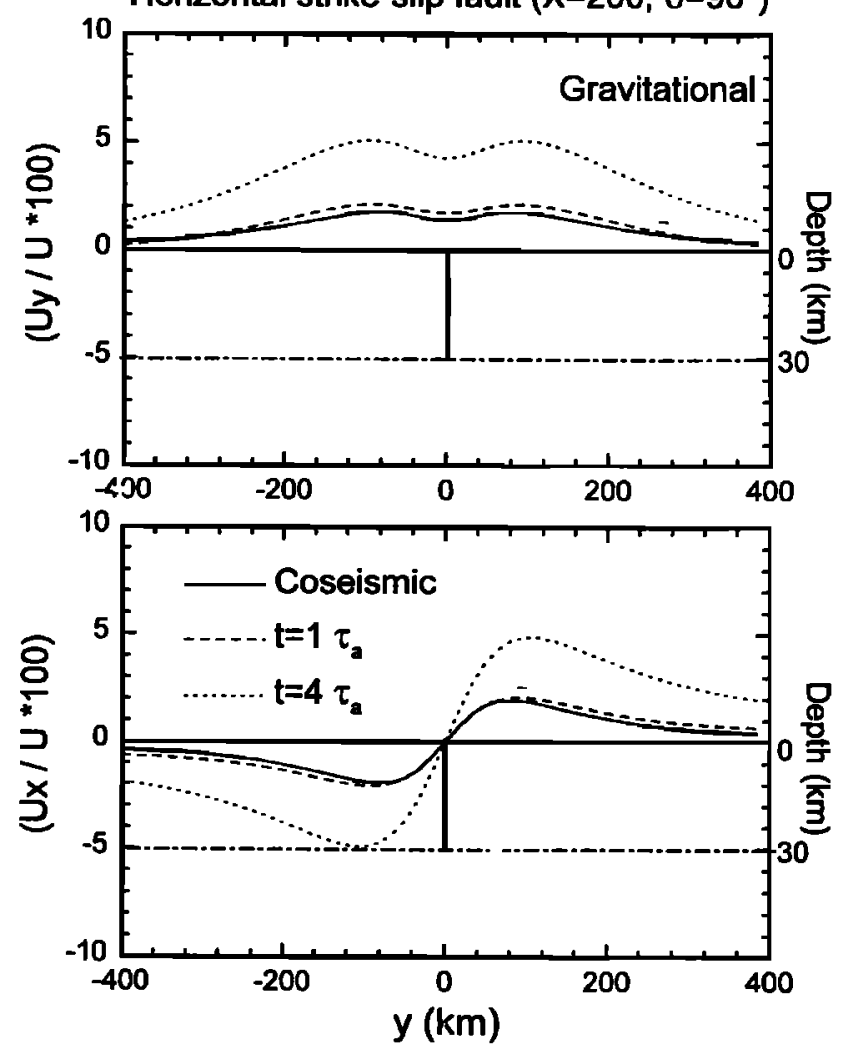

Figure 2. Surface horizontal deformation against distance normal to a strike-slip fault ruptures the entire elastic layer. Model parameters are $H=30 \mathrm{~km}, 2 L=200 \mathrm{~km}, \rho_{l}=\rho_{h}=3.0 \mathrm{~g} / \mathrm{cm}^{3}$, $\mu_{l}=\mu_{h}=\lambda_{1}=\lambda_{\mathrm{h}}=3 \times 10^{10} \mathrm{~Pa}$ (subscript $l$ denotes the elastic layer and subscript $h$ denotes the viscoelastic half-space), $D / H=0$, $W / H=1, \psi=90^{\circ}$. The coseismic response is calculated using an elastic half-space where $\lambda=\mu=3 \times 10^{10} \mathrm{~Pa}$. The horizontal dashed line is the elastic layered half-space boundary, and the thick vertical line represents the fault plane geometry. The solid curve is the initial elastic responses, and the dashed curves represent the deformation due to viscoelastic stress relaxation after $1 \tau_{\mathrm{a}}$ and $4 \tau_{\mathrm{a}}$ Each displacement profile has been evaluated at $100 \mathrm{~km}$ away from the end of the fault tip $(X=200 \mathrm{~km})$. Replacement for Figure 2 of $\mathrm{Yu}$ et al. 
Horizontal strike-slip fault $\left(X=200, \theta=90^{\circ}\right)$
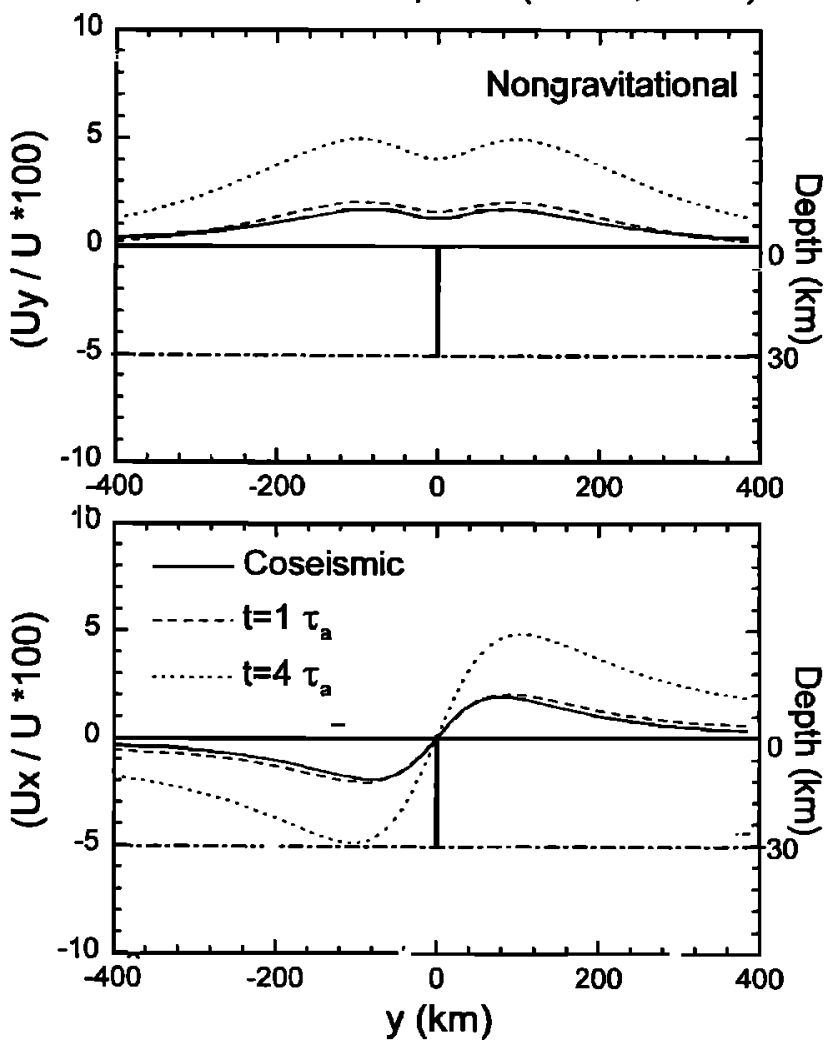

Figure 3. Surface horizontal deformation for vertical strike-slip fault without gravitational effect; the rest of the parameters are the same as Figure 2. Replacement for Figure 3 of Yu et al.

Horizontal strike-slip fault $\left(X=200, \theta=90^{\circ}\right)$
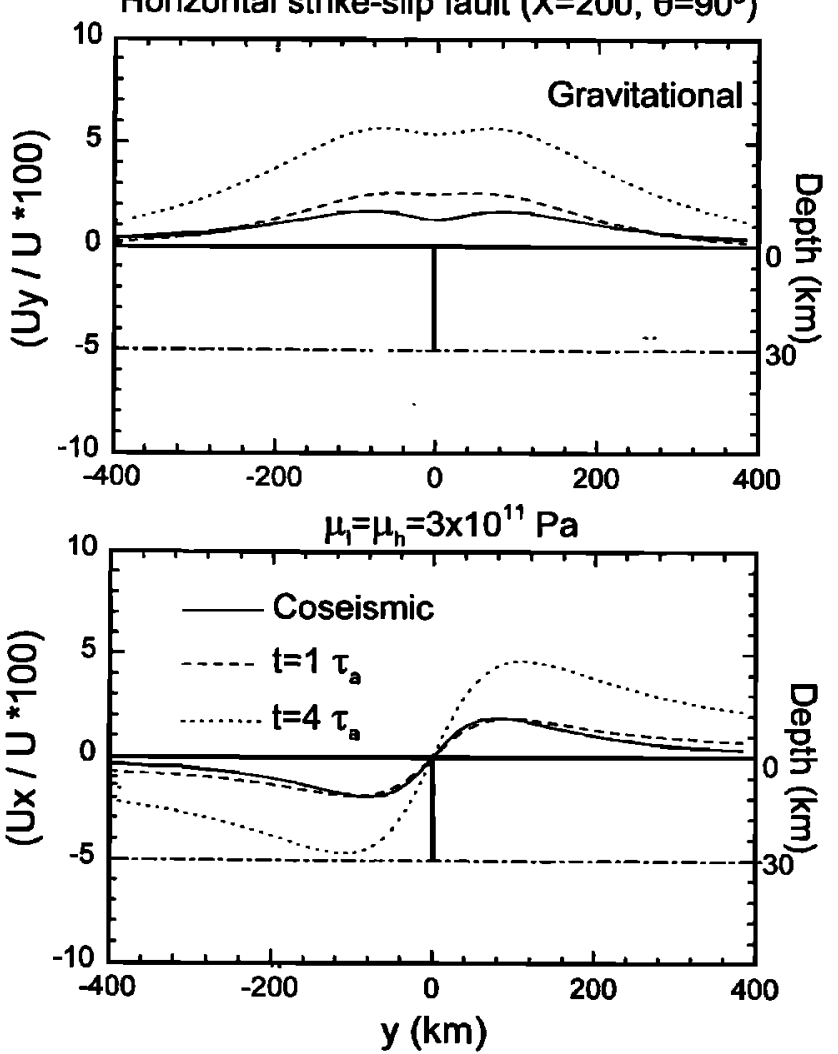

Figure 4. Replacement for Figure 4 of Yu et al.
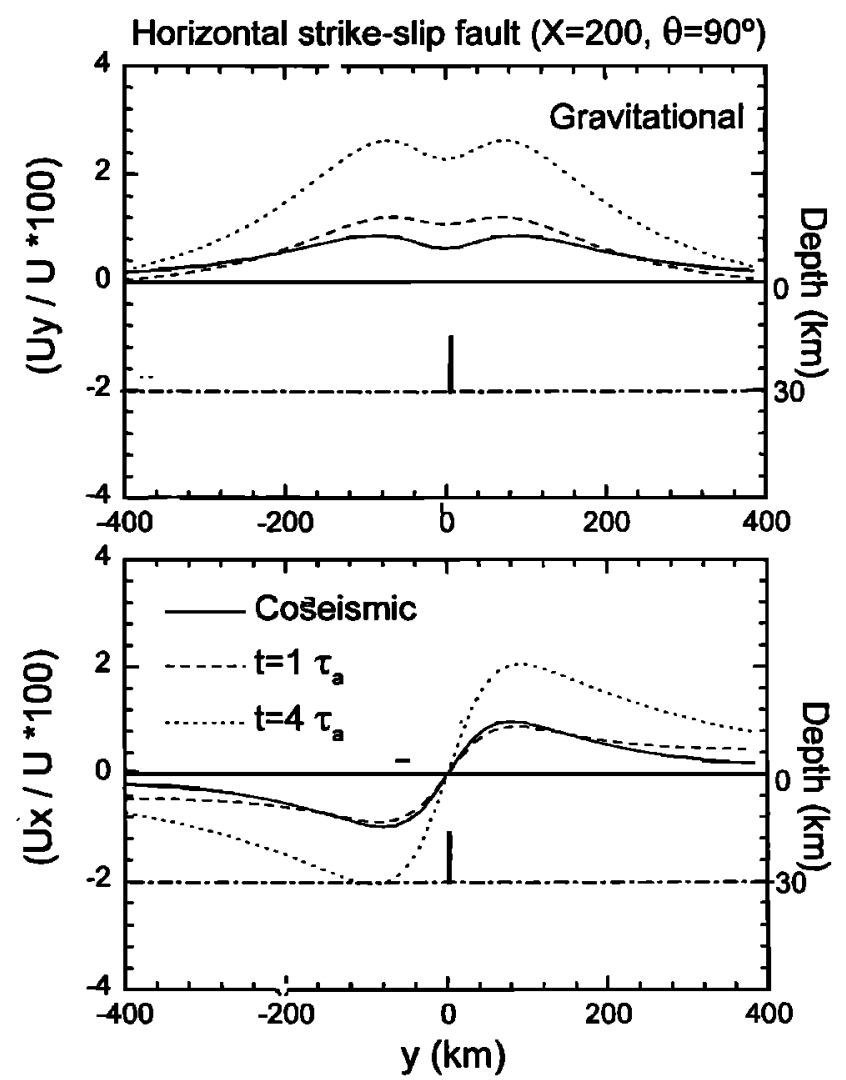

Figure 5. Surface horizontal deformations for a buried vertical strike slip fault, $D / H=0.5$, and $W / H=0.5$. ( $D$ is the depth of fault, $H$ is the thickness of elastic layer, and $W$ is the width of the fault.) Replacement for Figure 5 of Yu et al.
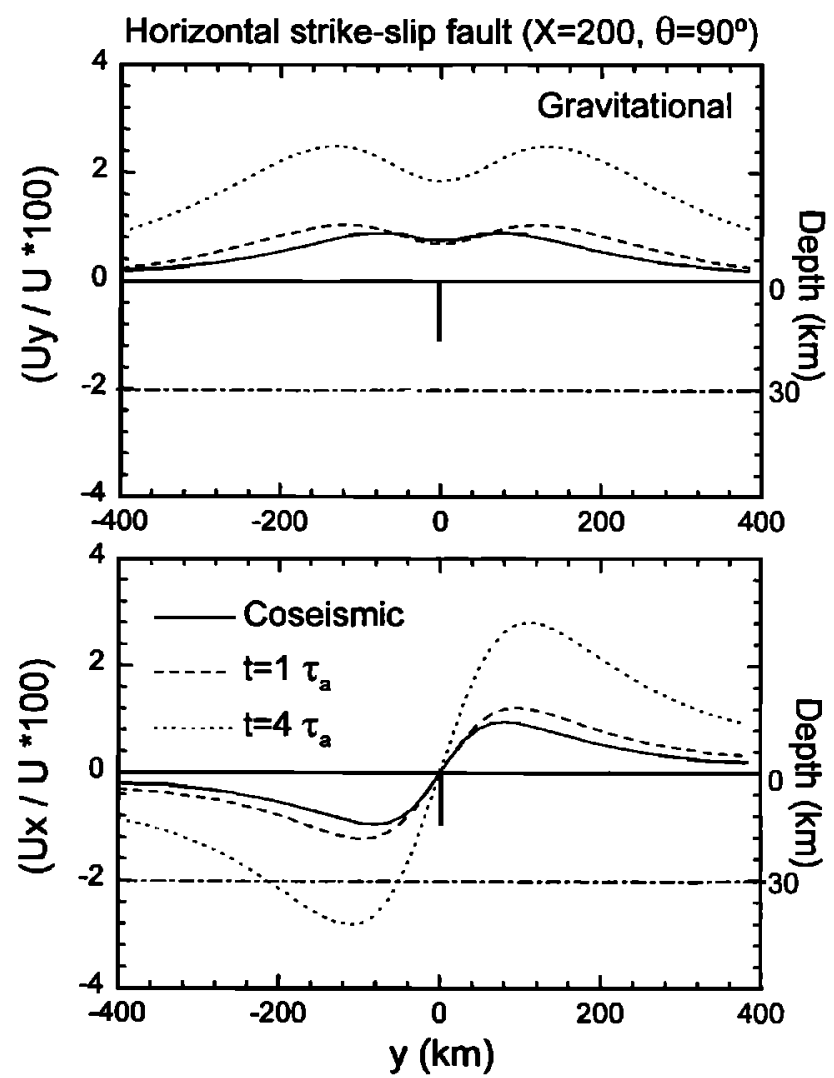

Figure 6. Surface horizontal deformations for a surface vertical strike-slip fault ruptures the top half of the elastic layer, $D / H=0$, and $W / H=0.5$. Replacement for Figure 6 of Yu et al. 
Horizontal strike-slip fault $\left(X=200, \theta=30^{\circ}\right)$
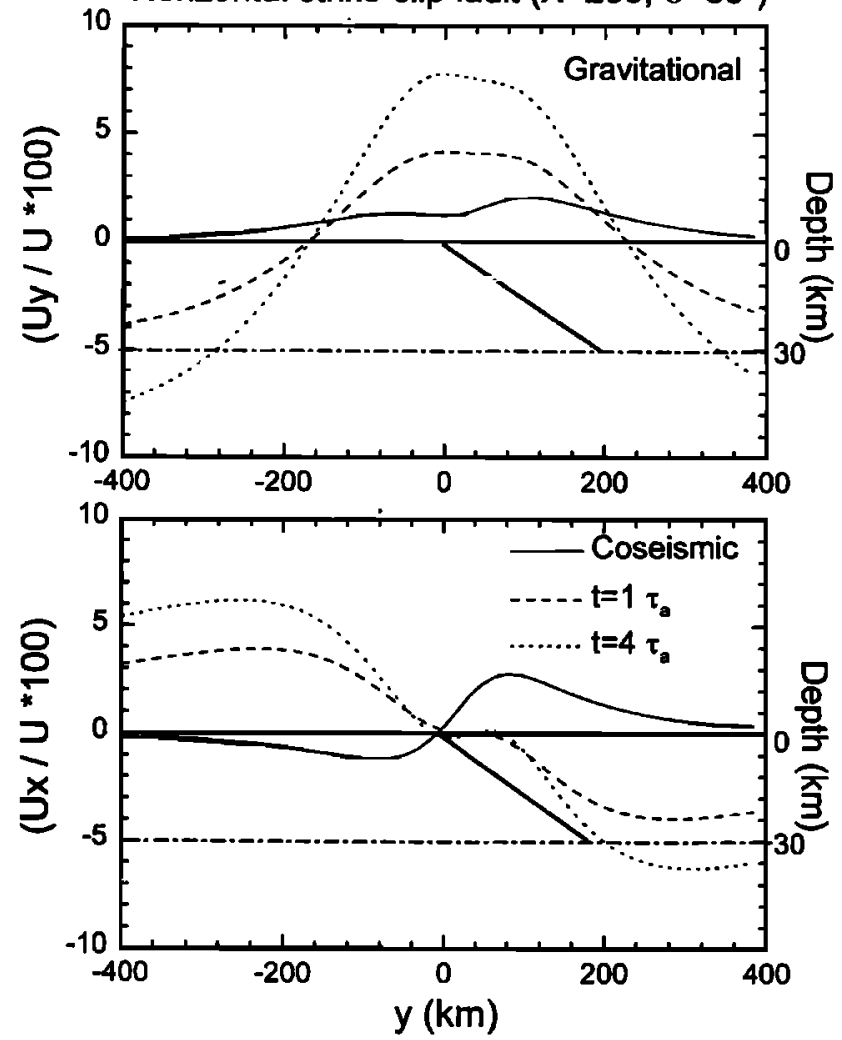

Figure 7. Surface horizontal deformations for dipping strike-slip fault, $\psi=30^{\circ}, D / H=0, W / H=1$. Replacement for Figure 7 of Yu et al.

$$
\begin{gathered}
u_{r}=\int_{0}^{\infty}\left\{\left[\frac{1}{i} y_{1}^{1}(0)\left(\frac{\partial}{\partial k r}\right) J_{1}(k r)+z_{1}^{1}(0)\left(\frac{1}{k r}\right) J_{1}(k r)\right] \cos \theta \cos \Psi\right. \\
\left.-\left[y_{2}^{1}(0)\left(\frac{\partial}{\partial k r}\right) J_{2}(k r)+\frac{1}{i} z_{2}^{1}(0)\left(\frac{1}{k r}\right) J_{2}(k r)\right] \sin 2 \theta \sin \Psi\right\} k d k
\end{gathered}
$$

Also, Figure 3 of $\mathrm{Yu}$ et al. is not correct for coseismic and postseismic displacements. The corresponding new Figure 3 is the correct one for both types of displacements. We observe that both new Figures 2 and 3 are really quite equal. To see clearly the differences between purely viscoelastic and viscoelasticgravitational postseismic displacements, we include Figure 9. The

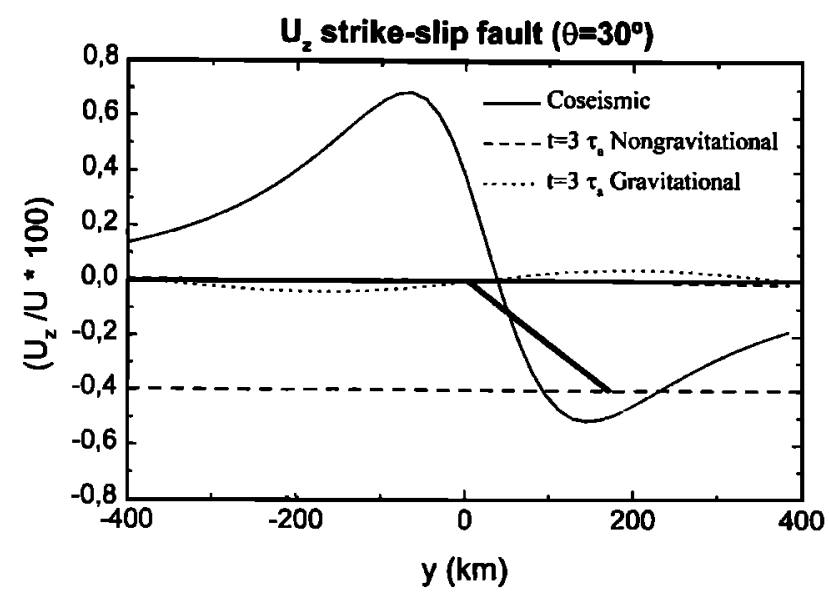

Figure 8. The vertical displacement due to a $30^{\circ}$ dipping strikeslip fault. Both the postseismic displacements with and without gravity are shown. Replacement for Figure 9 of Yu et al.
Horizontal strike-slip fault $\left(X=200, \theta=90^{\circ}\right)$
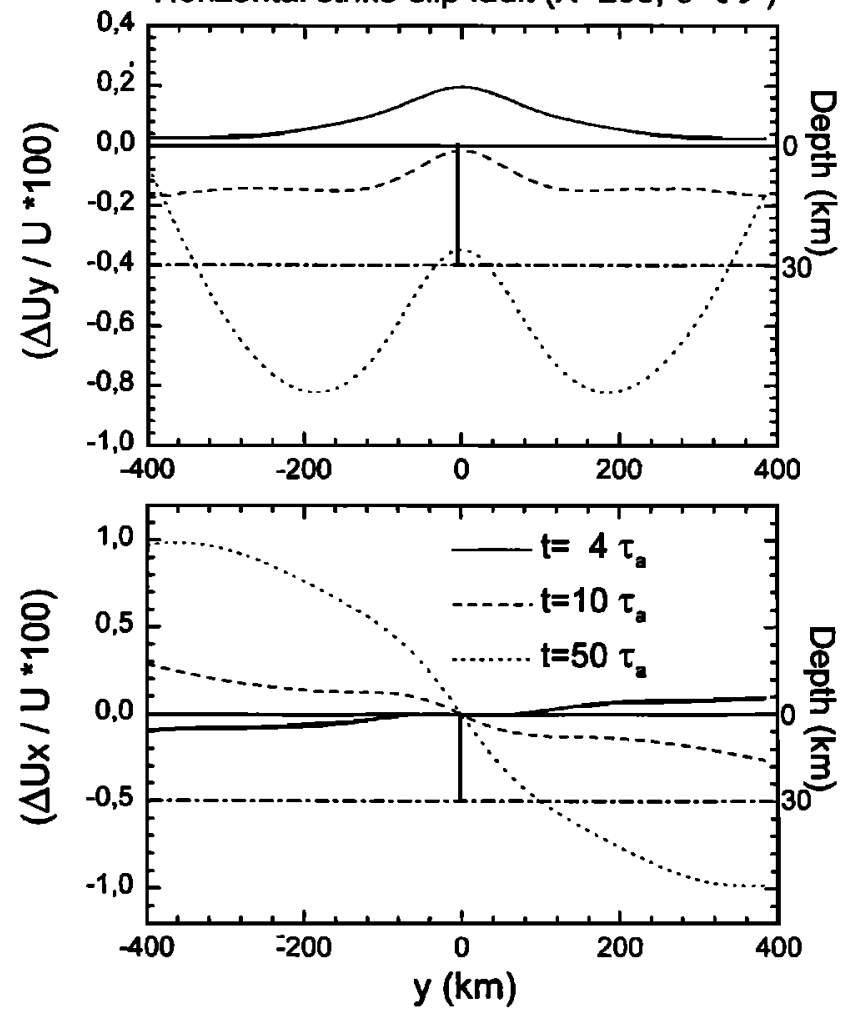

Figure 9. Viscoelastic-gravitational minus purely viscoelastic displacements $\left(\Delta U_{x}\right.$ and $\left.\Delta U_{y}\right)$ displacements, divided by dislocation $U$, for $X=200 \mathrm{~km}$ and the same fault as in Figure 2. In this figure, 4, 10 , and $50 \tau_{\mathrm{a}}$ are considered to show clearly the differences that appear after long periods of time.

results shown in Figure 9 are totally in agreement with the results obtained by Rundle [1981, 1982] and Fernández et al. [1996a, b].

Acknowledgments. Research by T.-T. Y. has been funded by the National Science Council under grant NSC88-2116-M-001-002. The research of J. B. R. has been supported under U. S. Department of Energy grant DE-FG03-95ER14499 to the Cooperative Institute for Research in Environmental Sciences (CIRES) at the University of Colorado at Boulder, and the research by J. F. has been supported by contracts AMB96-0498-C04-04 to the Institute of Astronomy and Geodesy, Madrid, and DE-FG03-95ER14499 to CIRES.

\section{References}

Femández, J., T.-T. Yu, and J.B. Rundle, Horizontal viscoelasticgravitational displacement due to a rectangular dipping thrust fault in a layered Earth model, J. Geophys. Res., 101, 13581-13594, 1996a (Correction, J. Geophys. Res., 103, 30283-30286, 1998.)

Femández, J., T.-T. Yu, and J.B. Rundle, Deformation produced by a rectangular dipping fault in a viscoelastic-gravitational layered Earth model, Part I, Thrust fault-fitgrv and fltgrh FORTRAN programs, Comput. Geosci., 22, 735-750, 1996b. (Cornection, Comput. Geosci., 25, 301-307, 1999.)

Okada, Y., Surface deformation due to shear and tensile faults in a halfspace, Bull. Seismol. Soc. Am., 75, 1135-1154, 1985.

Rundle, J.B., Numerical evaluation of static elastic gravitational deformation in a layered half-space by point couple sources, Rep. 81-2058, Sandia Natl. Lab., Albuquerque, N.M., 1981.

Rundle, J.B., Viscoelastic-gravitational deformation by a rectangular thrust fault in a layered Earth, J. Geophys. Res., 87, 7787-7796, 1982.

Yu, T.-T., J.B. Rundle, and J. Femández, Deformation produced by a rectangular dipping fault in a viscoelastic-gravitational layered Earth model, Part II, Strike-slip fault-strgrv and strgm FORTRAN programs. Comput. Geosci., 22, 751-764, 1996.

(Received March 11, 1999.) 